Cerebrovasc Dis 2007;24:319

DOI: $10.1159 / 000106520$

\section{Mild Mechanical Traumas Are Possible Risk Factors for Cervical Artery Dissection}

Sidney Rubinstein ${ }^{a}$, Pierre Côtéb

anstitute for Research in Extramural Medicine, VU University Medical Center, Amsterdam, The Netherlands; ${ }^{b}$ University Health Network Rehabilitation Solutions, Toronto Western Hospital, and Department of Public Health Sciences, Faculty of Medicine, University of Toronto, Toronto, Ont., Canada

The recent article by Dittrich et al. [1] provides important clinical information on the role of trivial trauma as a trigger of cervical artery dissection. However, we question the validity of the results regarding cervical manipulative therapy (CMT). Our principal objections concern three methodological issues, two of which are often inherent in observational studies: selection bias, confounding by indication, and imprecision surrounding the effect estimates.

Firstly, the use of nondissecting ischemic stroke as a control potentially introduced selection bias. Controls must be a representative sample of the study population, and have an equal chance to develop the target disease as the cases. If not, this could lead to an overestimation of risk. The mechanism of cerebral ischemia from nondissecting stroke is clearly different from dissection, and as one might suspect, the risk factors also differ [2]. A suitable control would, therefore, be a random, age- and sexmatched sample from the source population, thus providing a representative mix of various risk factors, including comorbidity.

Selection bias might, however, also arise in another manner; namely, it has been demonstrated that patients with a greater incidence of comorbidity are less likely to consult a chiropractor [3]. In this study, the controls had greater comorbidity, which (by design) would result in less exposure among the controls, and also inflate the estimate of risk.

Secondly, the authors do not consider the possibility of confounding by indication. It is well known that neck pain and headache are symptoms of a dissection in progress, even in the absence of frank neurological signs or symptoms [4-7]. These same symptoms might motivate patients to seek manipulative care of the neck, thinking that they are experiencing a musculoskeletal complaint. Thus, it is possible that in some cases, the manipulative treatment occurred following a developing stroke. Support for this argument is provided by the authors themselves. In table 1, all 4 categories of neck pain and headache prior to the onset of symptoms were much more prevalent among the cases than the controls.

Lastly, the adjusted odds ratios (ORs) provided in table 3 suggest a small increased risk for CMT. However, a close examination of these estimates raises two additional concerns. The crude and adjusted ORs are clearly different in magnitude, indicating that controlling for confounders significantly reduces the size of the observed effect. This suggests that residual confounding is likely present and that a fully adjusted OR would likely migrate even closer towards an $\mathrm{OR}=1$. For example, the residual confounding effects of smoking, alcohol consumption, or oral contraceptive use may explain the observed increase in risk. Additionally, the results presented in table 3 suffer from a gross lack of precision. The width of the confidence interval makes the point estimates difficult to interpret. For example, the results for CMT $<7$ days suggests that being exposed to cervical manipulative therapy may have an effect that ranges from a $70 \%$ decrease in risk (protective effect) to a $690 \%$ increase in risk.

While we recognize the methodological difficulties in conducting epidemiological studies on cervical artery dissection, we urge that these results be interpreted with caution and objectivity.

\section{References}

1 Dittrich R, Rohsbach D, Heidbreder A, Heuschmann P, Nassenstein I, Bachmann R, et al: Mild mechanical traumas are possible risk factors for cervical artery dissection. Cerebrovasc Dis 2006;23:275-281.

2 Rubinstein SM, Peerdeman SM, van Tulder MW, Riphagen I, Haldeman S: A systematic review of the risk factors for cervical artery dissection. Stroke 2005;36:1575-1580.

3 Cote P, Cassidy JD, Carroll L: The treatment of neck and low back pain: Who seeks care? Who goes where? Med Care 2001;39:956-967.

4 Dodick D: Headache as a symptom of ominous disease. What are the warning signals? Postgrad Med 1997;101:46-46, 62.

5 Lanfranchi S, Di Falco M, Perini M, Zarcone D: Posterior headache as a warning symptom of vertebral dissection: a case report. J Headache Pain 2005;6:478-479.

6 Mathys J, Lachat M, Herren T: Headache as a manifestation of a lifethreatening vascular disorder. Headache 2004;44:706-709.

7 Silbert PL, Mokri B, Schievink WI: Headache and neck pain in spontaneous internal carotid and vertebral artery dissections. Neurology 1995;45:1517-1522.

Sidney Rubinstein, DC, MSc

Institute for Research in Extramural Medicine (EMGO Institute)

VU University Medical Center, van der Boechorststraat 7

NL-1081 BT Amsterdam (The Netherlands)

Tel. +31 20444 8406, Fax +31 204446775

E-Mail SM.Rubinstein@vumc.nl 